\title{
Moment-Rotation Behavior of Force-Based Plastic Hinge Elements
}

\author{
Michael H. Scott ${ }^{\text {a) }}$ and Keri L. Ryan ${ }^{\text {b) }}$
}

The moment-rotation behavior of force-based frame elements is expressed as a function of plastic hinge length and moment-curvature parameters for two types of plastic hinge integration under the representative loading condition of antisymmetric bending. For modified Gauss-Radau hinge integration, there is a unique relationship between the resulting moment-rotation hardening ratio and parameters defining the plastic hinge length and moment-curvature hardening ratio. For two-point Gauss-Radau hinge integration, the spread of yielding across the hinge regions leads to a multilinear moment-rotation response, for which a secant approximation of the hardening stiffness is directed to a target plastic rotation. An example application demonstrates that significantly unconservative assessments of lateral load-carrying capacity can be attained if modeling parameters for plastic hinge length and moment-curvature strain hardening are not calibrated to account for the discrepancy between moment-curvature and moment-rotation behavior of an element. [DOI: 10.1193/1.4000136]

\section{INTRODUCTION}

Modeling structural resistance to extreme loads requires nonlinear frame finite elements that are well documented, simple to use, and computationally efficient. Due to their simplicity in simulating nonlinear flexural response, concentrated plasticity models are frequently used for capacity assessment, as in the pushover procedures outlined in ATC-55 (Applied Technology Council 2005). In the concentrated plasticity approach, nonlinear zero-length rotational springs are assembled at the ends of a linear-elastic element. Compatibility and equilibrium for the element are satisfied using either a two-component (Clough et al. 1965) or onecomponent (Giberson 1967) formulation. Concentrated plasticity models are well suited for parametric studies that desire a straightforward variation of basic structure properties (Fajfar et al. 2006, Alimoradi et al. 2007). Several researchers have used concentrated plasticity models to estimate seismic demand parameters for a comprehensive range of ground motions and structure characteristics (Ibarra et al. 2005, Medina and Krawinkler 2005, Goel 2005, Karavasilis et al. 2008). Detailed comparisons of concentrated plasticity formulations with varying levels of modeling complexity are given by Dides and de la Llera (2005).

Finite-element formulations offer an alternative approach to concentrated plasticity by allowing plasticity to distribute along the element length. Force-based frame elements have been shown to be advantageous over displacement-based elements for material nonlinear frame analysis (Alemdar and White 2005, Calabrese et al. 2010). Force-based

a) Oregon State University, School of Civil and Construction Engineering, Corvallis, OR 97331

b) University of Nevada, Department of Civil and Environmental Engineering, Reno, NV 89557 
plastic hinge elements, which represent a compromise between concentrated and distributed plasticity formulations, have also been used successfully in simulating the seismic response of structures (e.g., Berry et al. 2008). Although these plastic hinge elements are easy to use, the relation between the section constitutive response, the assumed plastic hinge length, and the element-level flexural response has not been conveyed in the literature.

This paper addresses this modeling issue by expressing the moment-rotation response of force-based elements as a function of moment-curvature and plastic hinge length parameters for two common plastic hinge integration techniques. These expressions allow an analyst, for common loading conditions, to calibrate the response of force-based plastic hinge elements to a desired moment-rotation behavior. The consequences of failing to calibrate the models in earthquake engineering simulation are demonstrated through a representative example.

\section{FORCE-BASED FRAME ELEMENTS}

As outlined by Neuenhofer and Filippou (1997) and the references therein, force-based elements satisfy equilibrium in strong form. Element compatibility is based on the principle of virtual forces, where element deformations and flexibility are integrated numerically from section response. The section locations and their integration weights are defined by GaussLobatto quadrature, which allows for the spread of plasticity along the element. Recent extensions of the force-based formulation developed by Scott and Fenves (2006) allow an analyst to incorporate a physically significant plastic hinge length in the element state determination. Two such approaches to plastic hinge integration that maintain the theoretically exact solution for a prismatic, linear-elastic frame element are briefly summarized here.

\section{TWO-POINT GAUSS-RADAU INTEGRATION}

For two-point Gauss-Radau integration, isoparametric locations $\{0,2 / 3\}$ and weights $\{1 / 4,3 / 4\}$ are mapped onto user-defined plastic hinge regions of length $l_{p I}$ and $l_{p J}$ at the element ends. This leads to the following section locations, $x$, and weights, $w$, in the plastic hinge regions:

$$
\begin{gathered}
x=\left\{0,2 l_{p I} / 3, L-2 l_{p J} / 3, L\right\} \\
w=\left\{l_{p I} / 4,3 l_{p I} / 4,3 l_{p J} / 4, l_{p J} / 4\right\}
\end{gathered}
$$

With this approach, plasticity can spread across two integration points in each hinge region with linear-elastic behavior assumed for the element interior, as shown in Figure 1a.

\section{MODIFIED GAUSS-RADAU INTEGRATION}

For modified Gauss-Radau integration, the mapping in Equations 1 and 2 is scaled by a factor of four so that the end weights are equal to the plastic hinge lengths $l_{p I}$ and $l_{p J}$. The section locations are relocated accordingly along the element:

$$
x=\left\{0,8 l_{p I} / 3, L-8 l_{p J} / 3, L\right\}
$$


(a) Two-Point Gauss-Radau

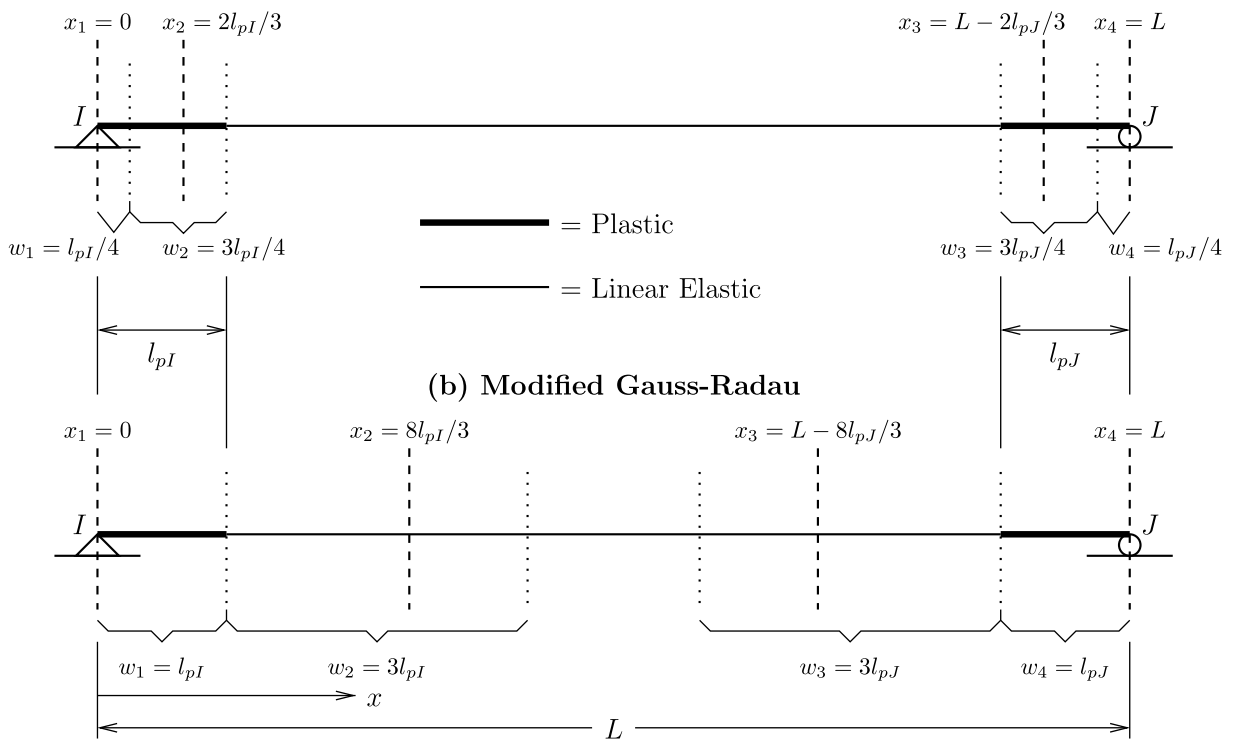

Figure 1. Section locations and weights for two types of plastic hinge integration: (a) two-point Gauss-Radau; (b) modified Gauss-Radau.

$$
w=\left\{l_{p I}, 3 l_{p I}, 3 l_{p J}, l_{p J}\right\}
$$

As shown in Figure 1b, plasticity is confined to the integration points at the element ends and linear-elastic response is imposed at the points on the element interior.

\section{MOMENT-ROTATION RESPONSE FOR ANTISYMMETRIC BENDING}

To express force-based element moment-rotation response in terms of plastic hinge length and section constitutive response, the beams shown in Figure 1 are loaded in a state of antisymmetric bending. The hinge lengths at the element ends are of equal length and defined by a ratio, $\beta$, of the element length, that is, $l_{p I}=l_{p J}=\beta L$. A bilinear momentcurvature relationship is prescribed for the plastic hinge regions with ratio of post-yield to initial stiffness equal to $\alpha$.

Under antisymmetric bending, the end moment-end rotation stiffness of a prismatic beam element in the linear-elastic range of response is $6 E I / L$. The ratio of post-yield to elastic stiffness is then defined as

$$
s=\frac{\Delta M}{\Delta \theta} \frac{L}{6 E I}
$$


Expressions are presented in the remainder of this section to show how the momentrotation hardening ratio depends on the plastic hinge length ratio, $\beta$, and moment-curvature hardening ratio, $\alpha$, in a force-based plastic hinge element loaded in antisymmetric bending.

\section{MODIFIED GAUSS-RADAU INTEGRATION}

With modified Gauss-Radau integration, yielding is confined to the integration points at the element ends. As a result, the element moment-rotation response takes on the characteristics of the section response, as shown in Figure 2a for antisymmetric bending with the assumed bilinear moment-curvature behavior. Using the principle of virtual forces, the post-yield moment-rotation response can be calculated as

$$
\Delta \theta=\left(\frac{\beta}{\alpha}+3 \beta\left(1-\frac{16 \beta}{3}\right)^{2}+\frac{(1-8 \beta)^{3}}{6}\right) \frac{\Delta M L}{E I}
$$

Full details of this calculation are shown in Scott (2004). After algebraic simplification, the moment-rotation hardening ratio defined in Equation 5 is obtained from the previous equation as a function of $\alpha$ and $\beta$

$$
s=\frac{\alpha}{\alpha+(1-\alpha) 6 \beta}
$$

Over the range $\beta=[0,0.5], s$ is inversely proportional to $\beta$ with corresponding values of $s$ ranging from 1 to $\alpha /(3-2 \alpha)$, as shown in Figure $2 \mathrm{~b}$. Note that when the hinge length parameter $\beta$ is equal to $1 / 6$, the hardening ratios for moment-curvature and moment-rotation response are equal, that is, $\alpha=s$. Furthermore, depending on available modeling data, Equation 7 can be rearranged to solve for $\beta$ as a function of $s$ and $\alpha$, or for $\alpha$ as a function of $s$ and $\beta$.

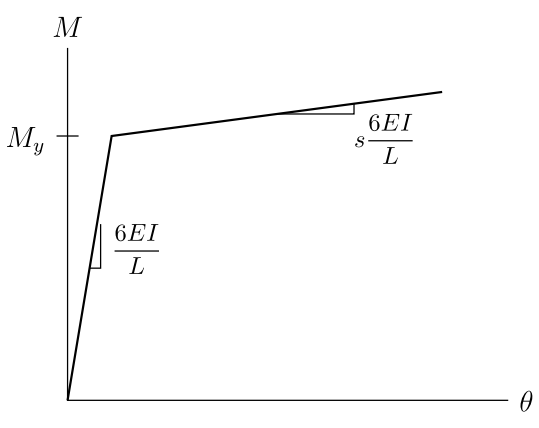

(a)

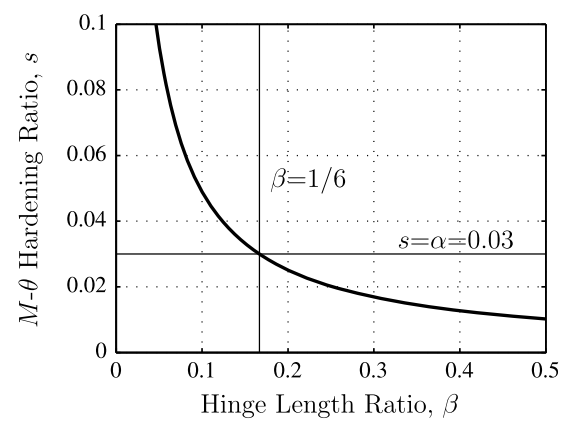

(b)

Figure 2. (a) Moment-rotation behavior of modified Gauss-Radau element under antisymmetric bending; (b) moment-rotation hardening ratio as a function of hinge ratio for $\alpha=0.03$. 


\section{TWO-POINT GAUSS-RADAU INTEGRATION}

For bilinear moment-curvature response and two-point Gauss-Radau in the plastic hinge regions, the element moment-rotation response under antisymmetric bending is trilinear, as shown in Figure 3. Distinct moment-rotation hardening ratios, $s_{1}$ and $s_{2}$, correspond to the initiation of yielding at the first and second hinge integration points, respectively. With calculations similar to those used in obtaining Equation 7, expressions for $s_{1}$ and $s_{2}$ are

$$
\begin{gathered}
s_{1}=\frac{\alpha}{\alpha+(1-\alpha)(3 \beta / 2)} \\
s_{2}=\frac{\alpha}{\alpha+(1-\alpha)\left(6 \beta-12 \beta^{2}+8 \beta^{3}\right)}
\end{gathered}
$$

The two-point Gauss-Radau response can be calibrated to a secant post-yield approximation directed to a target plastic rotation, $\theta_{p}$. Using the geometry of Figure 3 , the secant stiffness can be expressed as

$$
s=\frac{s_{1} \theta^{*}+s_{2}\left(\theta_{p}-\theta^{*}\right)}{\theta_{p}}
$$

where $\theta^{*}$ is the change in rotation as yielding spreads from the first to second integration point in the plastic hinge regions

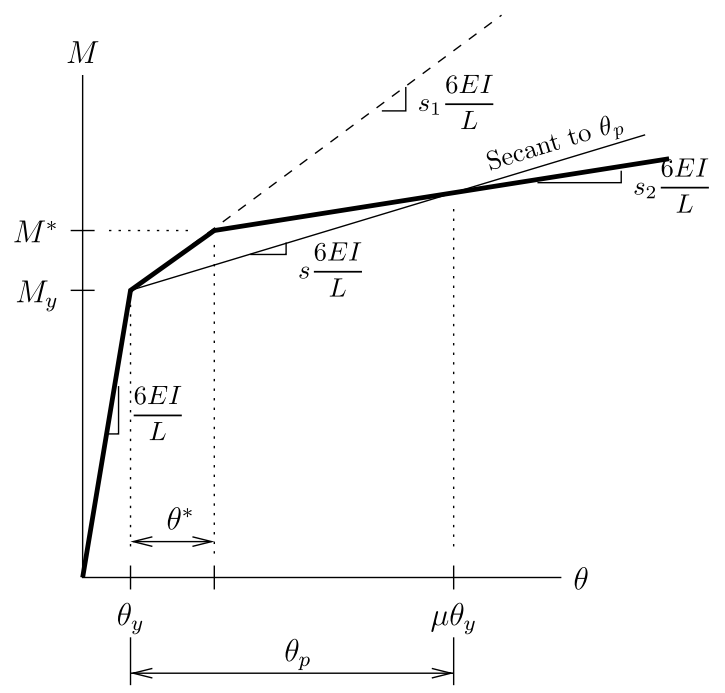

Figure 3. Bilinear secant approximation of post-yield response of a force-based element with two-point Gauss-Radau plastic hinge integration under antisymmetric bending. 


$$
\theta^{*}=\frac{L}{6 E I}\left(\frac{M^{*}-M_{y}}{s_{1}}\right)
$$

Based on equilibrium under antisymmetric bending, it is straightforward to show that the end moment when yielding initiates at the second integration point is $M^{*}=M_{y} /(1-4 \beta / 3)$ with the integration points defined in Equation 1. Accordingly, the change in end rotation can be expressed as

$$
\theta^{*}=\left(\frac{4 \beta / 3}{1-4 \beta / 3}\right) \frac{\theta_{y}}{s_{1}}
$$

Substituting this value into Equation 10 and using the rotation ductility shown in Figure 3, $\mu=1+\theta_{p} / \theta_{y}$, gives the secant hardening ratio

$$
s=s_{2}+\frac{4 \beta / 3}{1-4 \beta / 3}\left(1-\frac{s_{2}}{s_{1}}\right) \frac{1}{\mu-1}
$$

To achieve a desired secant hardening ratio for a target rotation ductility, one of $\alpha$ or $\beta$ should be selected and the other computed by solving Equation 13. However, the secant approximation is feasible only if $s_{1}$ computed in Equation 8 is greater than the target hardening ratio, $s$.

The solution to Equation 13 for $\alpha=0.03$ and several rotation ductilities is shown in Figure $4 \mathrm{a}$ over a range of hinge length ratios that reveals distinct features of the secant approximation. The secant approximation has a local minimum in the feasible region, making it impossible to achieve a moment-rotation hardening ratio that is equal to the moment-curvature hardening ratio, except for cases where both the hinge length and rotation

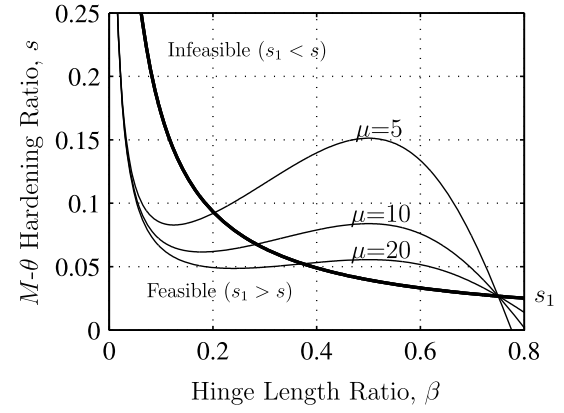

(a)

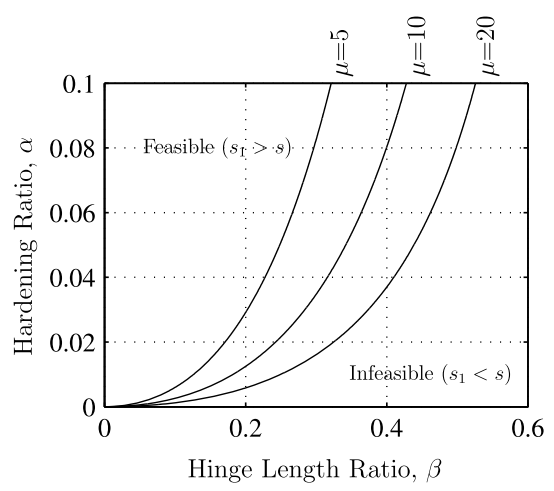

(b)

Figure 4. Secant approximation to moment-rotation hardening ratio under antisymmetric bending of two-point Gauss-Radau plastic hinge integration: (a) feasibility condition for $\alpha=0.03$; (b) combinations of $\alpha$ and $\beta$ leading to a feasible secant post-yield stiffness. 
ductility are unrealistically large. Another interpretation of the feasibility condition is shown in Figure $4 \mathrm{~b}$, where feasible combinations of $\alpha$ and $\beta$ lie to the left of each ductility curve. This figure shows that for a given $\alpha$, the hinge length ratio is constrained to a smaller fraction of the total element length as the target rotation ductility is decreased.

\section{EXAMPLE APPLICATION}

A single-bay three-story frame with uniform stiffness and strength over its height is used to illustrate the use of force-based elements with both forms of Gauss-Radau plastic hinge integration calibrated for specified moment-rotation behavior. In addition to the features shown in Figure 5, the frame has the following characteristics:

- A dead load of $889.6 \mathrm{kN}$ (200 kips) is applied at each story, giving a total structure weight, $W$, of 2,669 kN (600 kips).

- The yield base shear coefficient is $V / W=0.4$.

- The flexural stiffness, EI, is identical for the beams and the columns at each level, with values given in Figure 5.

- $\quad$ Rotational springs at the base of the columns (stiffness $k_{r}=3 E I / L$ ) lead to a more natural distribution of stiffness over the height (Medina and Krawinkler 2005).

- $\quad$ Plastic hinges form at the beam ends (yield moment $M_{y b}$ ) and in the base rotational springs (yield moment $M_{y c}$ ), while the columns are assumed to remain elastic.

- Pushover analyses of the frame are conducted in the OpenSees framework (McKenna et al. 2000) using the forceBeamColumn element command with both versions of Gauss-Radau plastic hinge integration.

A bilinear moment-rotation response with post-yield hardening ratio $s=0.03$ is desired for the beams. A common misuse of plastic hinge elements, referred to as the "uncalibrated"

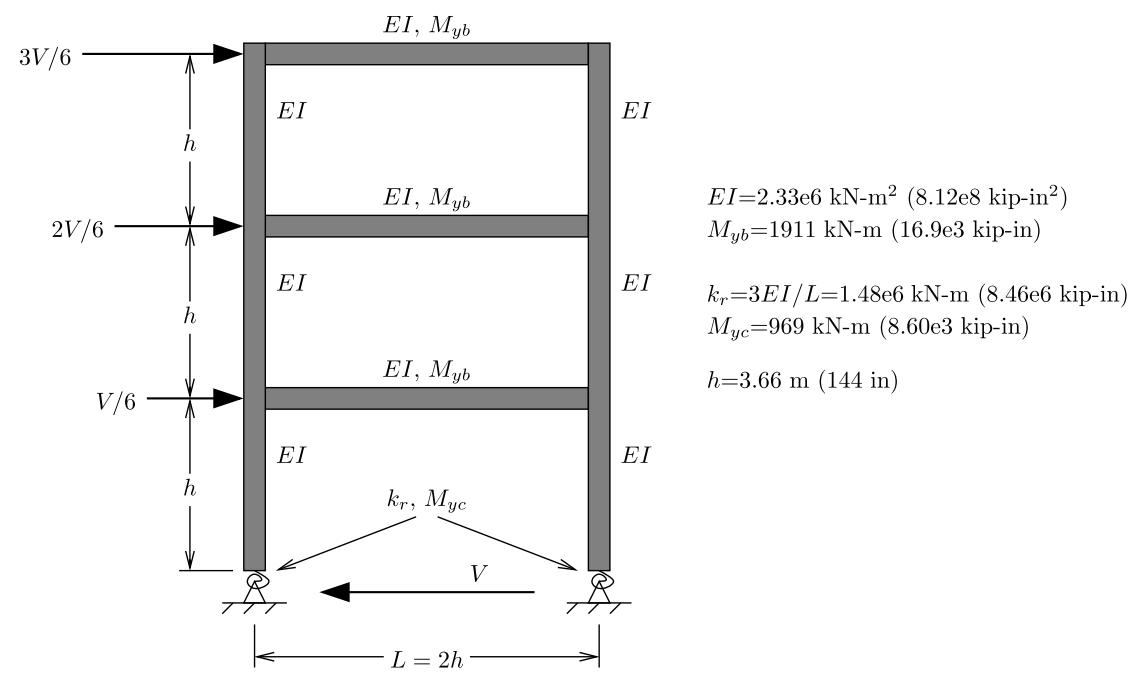

Figure 5. Example three-story frame used to demonstrate calibration procedures. 
case, is to select a plastic hinge length ratio based on member depth to span ratio, for example, $\beta=0.1$, and choose the moment-curvature hardening ratio to be equal to the desired moment-rotation hardening ratio, that is, $\alpha=s=0.03$. As shown in this example, the uncalibrated case can lead to post-yield response that is significantly stiffer than the desired response, which can be achieved by proper selection of the moment-curvature hardening ratio, $\alpha$, and plastic hinge length ratio, $\beta$.

\section{MODIFIED GAUSS-RADAU CALIBRATION}

With modified Gauss-Radau integration, any combination of $\alpha$ and $\beta$ that satisfies $s=$ 0.03 according to Equation 7 will achieve the desired moment-rotation response.

1. Assuming that the hardening ratio of the moment-curvature response is the same as the desired moment-rotation response, that is, $\alpha=s=0.03$, requires using a hinge length ratio of $\beta=1 / 6$.

2. Assuming a hinge length ratio of $\beta=0.1$ requires changing the moment-curvature hardening ratio to $\alpha=0.0182$.

The calibrated member and structural response based on either of the above listed approaches is shown in Figure 6 along with the uncalibrated response. As the roof drift exceeds $2 \%$, the base shear coefficient for the uncalibrated case is more than $20 \%$ higher than that obtained using calibrated parameters.

\section{TWO-POINT GAUSS-RADAU SECANT CALIBRATION}

Since the moment-rotation response using two-point Gauss-Radau hinge integration is trilinear, the desired bilinear moment-rotation response cannot be realized exactly. Instead, a secant approximation can be obtained for a target rotation ductility, $\mu$, and a combination of $\alpha$ and $\beta$ that satisfies the feasibility condition $s_{1}>s$. To illustrate the secant calibration, the target rotation ductility is assumed to $\mu=10$, making the plastic rotation $\theta_{p}=10 \theta_{y}=0.01$ rad. This represents a lower plastic rotation, but a higher ductility, than is typical due to the uncharacteristically large stiffness-to-strength ratios of the generic frame members.
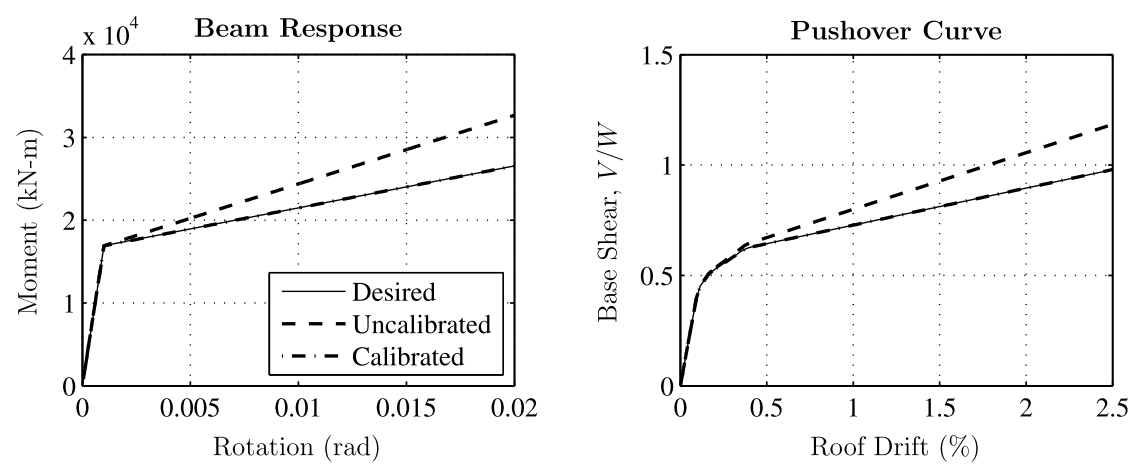

Figure 6. Building frame response with calibrated modified Gauss-Radau plastic hinge element. 
1. Assuming $\alpha=s$, it is not possible to find a feasible hinge length ratio $\beta$ to achieve $s=0.03$ for the target rotation ductility $\mu=10$. As shown in Figure $4 \mathrm{a}, s$ has a minimum value of about 0.06 over the range of realistic hinge length ratios $(\beta \leq 0.5)$ when $\mu=10$ and $\alpha=0.03$.

2. Assuming $\beta=0.1$, the moment-curvature hardening ratio is computed as $\alpha=0.00905$ from Equation 13 with $\mu=10$ in order to achieve $s=0.03$. For this combination of $\alpha$ and $\beta, s_{1}=0.0574$ according to Equation 8 , which satisfies the feasibility condition $s_{1}>s$. The computed response for this case is shown in Figure $7 \mathrm{a}$, along with the uncalibrated scenario, which leads to base shear that is $30 \%$ higher than that obtained with calibrated parameters.

3. Increasing the target rotation ductility to $\mu=20$ and keeping the assumed hinge length ratio $\beta=0.1$ requires increasing the moment-curvature hardening ratio to $\alpha=0.0121$ in order to achieve $s=0.03$. This also represents a feasible combination of $\alpha$ and $\beta$ as $s_{1}=0.0756>s$. The computed member and structural responses are shown in Figure $7 \mathrm{~b}$ for this case.
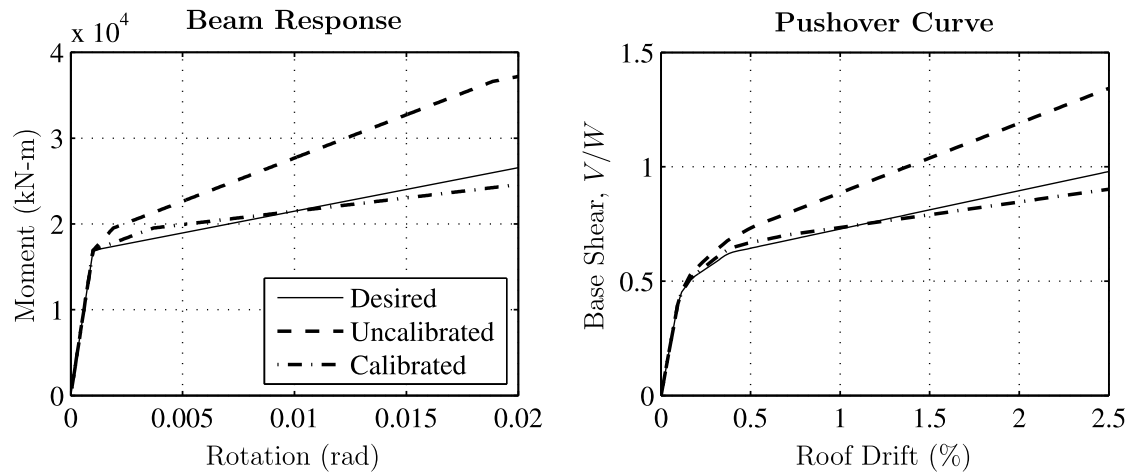

(a) $\mu=10, \beta=0.1 \longrightarrow \alpha=0.00905$
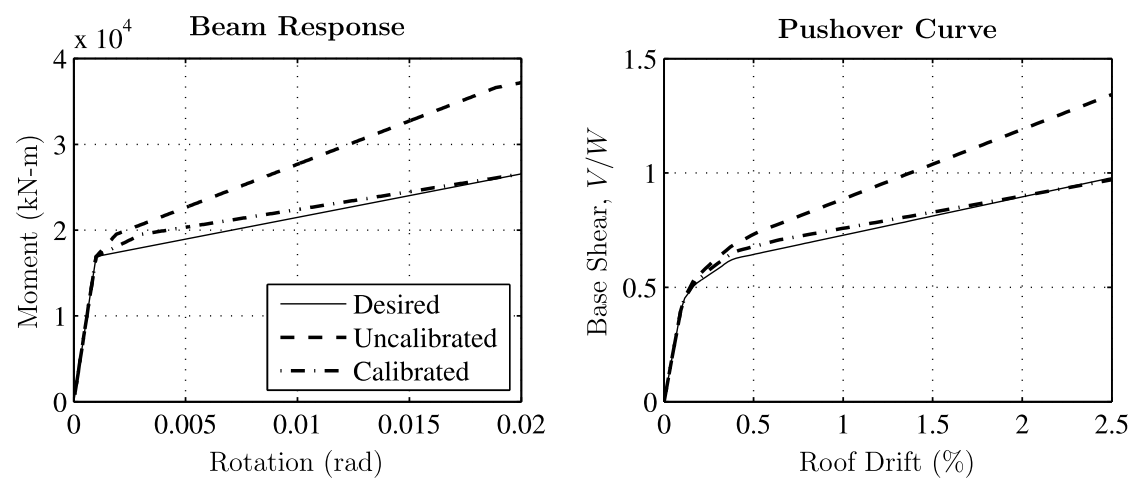

(b) $\mu=20, \beta=0.1 \longrightarrow \alpha=0.0121$

Figure 7. Building frame response with calibrated two-point Gauss-Radau plastic hinge element for target rotation ductility: (a) $\mu=10$; and (b) $\mu=20$. 


\section{CONCLUSIONS}

Two versions of Gauss-Radau hinge integration in force-based frame elements were examined for their moment-rotation response. The following modeling approaches achieve a desired moment-rotation response under antisymmetric bending with bilinear momentcurvature behavior:

- For modified Gauss-Radau plastic hinge integration, any combination of the moment-curvature hardening ratio, $\alpha$, and plastic hinge length ratio, $\beta$, that satisfies Equation 7 can be used to achieve a target bilinear moment-rotation behavior.

- When the plastic hinge length ratio is $\beta=1 / 6$ for modified Gauss-Radau integration, the moment-rotation response has the same post-yield stiffness ratio as that for the moment-curvature response in the plastic hinge regions.

- $\quad$ For two-point Gauss-Radau integration, first the moment-curvature parameter, $\alpha$, or the plastic hinge length ratio, $\beta$, should be chosen, then the other computed from Equation 13 for a prescribed moment-rotation response and target rotation ductility. Feasible combinations of $\alpha$ and $\beta$ depend on the target rotation ductility, as shown in Figure $4 b$.

Pushover analyses of a simplified frame show that lateral load capacity can be significantly overpredicted if moment-curvature or plastic hinge length parameters are not calibrated to account for the difference between the section moment-curvature and element moment-rotation response.

\section{REFERENCES}

Alemdar, B. N., and White, D. W., 2005. Displacement, flexibility, and mixed beam-column finite element formulations for distributed plasticity analysis, Journal of Structural Engineering 131, 1811-1819.

Alimoradi, A., Pezeshk, S., and Foley, C. M., 2007. Probabilistic performance-based optimal design of steel moment-resisting frames. II: Applications, Journal of Structural Engineering 133, 767-776.

Applied Technology Council, 2005. ATC-55: Evaluation and Improvement of Inelastic Seismic Analysis Procedures, Redwood City, CA.

Berry, M. P., Lehman, D. E., and Lowes, L. N., 2008. Lumped-plasticity models for performance simulation of bridge columns, ACI Structural Journal 105, 270-279.

Calabrese, A., Almeida, J. P., and Pinho, R., 2010. Numerical issues in distributed inelasticity modeling of RC frame elements for seismic analysis, Journal of Earthquake Engineering 14, $38-68$.

Clough, R. W., Benuska, K. L., and Wilson, E. L., 1965. Inelastic earthquake response of tall buildings, Third World Conference on Earthquake Engineering, Wellington, New Zealand.

Dides, M. A., and de la Llera, J. C., 2005. A comparative study of concentrated plasticity models in dynamic analysis of building structures, Earthquake Engineering and Structural Dynamics 34, 1005-1026.

Fajfar, P., Dolsek, M., Marusic, D., and Stratan, A., 2006. Pre- and post-test mathematical modeling of a plan-asymmetric reinforced concrete frame building, Earthquake Engineering and Structural Dynamics 35, 1359-1379. 
Giberson, M. F., 1967. The Response of Nonlinear Multistory Structures Subjected to Earthquake Excitation, Ph.D. Thesis, California Institute of Technology, Pasadena, CA.

Goel, R. K., 2005. Evaluation of modal and FEMA pushover procedures using strong-motion records of buildings, Earthquake Spectra 21, 653-684.

Ibarra, L. F., Medina, R. A., and Krawinkler, H., 2005. Hysteretic models that incorporate strength and stiffness deterioration, Earthquake Engineering and Structural Dynamics 34, $1489-1511$.

Karavasilis, T. L., Bazeos, N., and Beskos, D. E., 2008. Drift and ductility estimates in regular steel MRF subjected to ordinary ground motions: A design-oriented approach, Earthquake Spectra 24, 431-451.

McKenna, F., Fenves, G. L., and Scott, M. H., 2000. Open System for Earthquake Engineering Simulation, University of California, Berkeley, CA, http://opensees.berkeley.edu.

Medina, R. A., and Krawinkler, H., 2005. Evaluation of drift demands for the seismic performance assessment of frames, Journal of Structural Engineering 131, 1003-1013.

Neuenhofer, A., and Filippou, F. C., 1997. Evaluation of nonlinear frame finite-element models, Journal of Structural Engineering 123, 958-966.

Scott, M. H., 2004. Software Frameworks for the Computational Simulation of Structural Systems, Ph.D. Thesis, University of California, Berkeley, CA.

Scott, M. H., and Fenves, G. L., 2006. Plastic hinge integration methods for force-based beamcolumn elements, Journal of Structural Engineering 132, 244-252.

(Received 11 January 2011; accepted 10 March 2012) 